\title{
Prospective Study of the Surgical Techniques Used in Primary Rhinoplasty on the Caucasian Nose and Comparison of the Preoperative and Postoperative Anthropometric Nose Measurements
}

\author{
Cezar Augusto Sarraf Berger ${ }^{1,2,3}$ Renato da Silva Freitas ${ }^{4}$ Osvaldo Malafaia5,6 \\ José Simão de Paula Pinto ${ }^{7}$ Evaldo Dacheux Macedo Filho ${ }^{3,8}$ Marcos Mocellin ${ }^{3}$ \\ Marina Serrato Coelho Fagundes ${ }^{9}$
}

${ }^{1}$ Fellowship Program on Facial Plastic Surgery, IPO Hospital, Curitiba,

Address for correspondence Cezar Augusto Sarraf Berger, MD, MSc, Paraná, Brazil

2 MSc in Surgical Practice, Universidade Federal do Paraná (UFPR), Department of Otolaryngology, Hospital Paranaense de Otorrinolaringologia IPO, Av. República Argentina, 2069 Bairro Agua Curitiba, Paraná, Brazil

${ }^{3}$ Department of Otolaryngology, UFPR Clinical Hospital (HC/UFPR), Verde Curitiba, Paraná 80620010, Brazil (e-mail: nep@ipo.com.br).

Curitiba, Paraná, Brazil

${ }^{4}$ Chief of Plastic Surgery Procedure, UFPR, Curitiba, Paraná, Brazil

${ }^{5}$ Titular Professor of Surgery, UFPR, Curitiba, Paraná, Brazil

6 Institute of Medical Research and of the Postgraduate Program; MSc and PhD on Surgery Principles, Evangélico University Hospital,

Curitiba, Paraná, Brazil

${ }^{7}$ Department of Science and Information Management, UFPR, Curitiba, Paraná, Brazil

${ }^{8}$ Department of NEP, IPO Hospital, Curitiba, Paraná, Brazil

${ }^{9}$ Department of Otolaryngology, IPO Hospital, Curitiba, Paraná, Brazil

Int Arch Otorhinolaryngol 2015;19:34-41.

\begin{abstract}
Keywords

- rhinoplasty

- prospective studies

- measurements

Introduction The knowledge and study of surgical techniques and anthropometric measurements of the nose make possible a qualitative and quantitative analysis of surgical results.

Objective Study the main technique used in rhinoplasty on Caucasian noses and compare preoperative and postoperative anthropometric measurements of the nose. Methods A prospective study with 170 patients was performed at a private hospital. Data were collected using the Electronic System Integrated of Protocols software (Sistema Integrado de Protocolos Eletrônicos, SINPE@). The surgical techniques used in the nasal dorsum and tip were evaluated. Preoperative and 12-month follow-up photos as well as the measurements compared with the ideal aesthetic standard of a Caucasian nose were analyzed objectively. Student $t$ test and standard deviation test were applied. Results There was a predominance of endonasal access (94.4\%). The most common dorsum technique was hump removal (33.33\%), and the predominance of sutures
\end{abstract}

received

May 26, 2014

accepted after revision

August 13, 2014

published online

December 2, 2014
DOI http://dx.doi.org/

10.1055/s-0034-1393721. ISSN 1809-9777.
Copyright $(2015$ by Thieme Publicações License terms

Ltda, Rio de Janeiro, Brazil
()(1) $\Theta \circledast$ 
(24.76\%) was observed on the nasal tip, with the lateral intercrural the most frequent (32.39\%). Comparison between preoperative and postoperative photos found statistically significant alterations on the anthropometric measurements of the noses.

Conclusion The main surgical techniques on Caucasian noses were evaluated, and a great variety was found. The evaluation of anthropometric measurements of the nose proved the efficiency of the performed procedures.

\section{Introduction}

Jack Gunter in his classic book Dallas Rhinoplasty: Nasal Surgery by the Masters mentions that the access incisions in rhinoplasty should not be seen only as external or internal accesses. ${ }^{1}$ Every nasal access necessarily acts in specific areas of the nose with higher or lower trauma, exposition, possibility of complex surgical maneuvers, and use of grafts and/or sutures.

Several surgical maneuvers currently known in open rhinoplasty can be performed in closed rhinoplasty, which results in less morbidity, reduced surgery time, and predictable results. The knowledge and analysis of surgical techniques as well as the anthropometric measurements of the nose make possible the quantitative analysis of the results, which is of great importance for the surgeon and for the services regarding facial plastic surgery. The record of surgical techniques performed is important for the study of successes and the side effects of rhinoplasty.

High-quality clinical trials are essential for continuous scientific development. They allow safe access to new information resulting in improvement of knowledge, target planning, spread of evaluation of procedures, and professional conduct.

The Electronic System Integrated of Protocols (SINPE) is a computer program created for manage database, idealized in the beginning of the 1990s by Dr. Osvaldo Malafaia, whose purpose is the development of electronic protocols for fact gathering for its subsequent utilization in clinical study.

In addition to enabling the construction of protocols, SINPE also provides a data analysis module, which performs the descriptive statistical evaluation. This information visualization interface, called SINPE Analyzer, is able to generate graphs and statistics, save results, and export data. ${ }^{2}$

The present study aims to evaluate the main techniques used in rhinoplasty on Caucasian noses, comparing preoperative and postoperative anthropometric measurements of the nose.

\section{Methods}

This research was conducted in a private hospital. The work was approved by the ethics committee of the institution (2528.135/20116), and an explained and free consent form was provided to the patients.

Data collection was performed prospectively from February 2010 to March 2011 by the researchers, using the SINPE software. A specific protocol called Rhinoplasty was created based on 954 items, being part of the SINPE Analyzer module, which quickly gathers the information in the protocols given by the data collection. ${ }^{3,4}$ The generation and storage of graphs make possible a quick analysis of extensive protocols, and it is also possible to copy each graph to the analysis sheet and include comments and references, creating a sequence of selfanalysis. This protocol was developed by Dr. Cezar Berger in 2011 for his master's thesis. ${ }^{5}$

The SINPE Analyzer and statistical analysis (Student $t$ test) were used for the validation of the protocol through the analysis of the existing data in the database; $p<0.05$ was considered significant.

The research started with 170 patients who underwent surgery; 58 patients, however, were not present throughout the times evaluated, which makes 112 the total number of patients who participated in all the phases of the study.

The information was collected in four phases: (D1) immediately postoperative and at (D2) 3-month, (D3) 6-month, and (D4) 12-month follow-up. Patients' clinical conditions were followed according to the specific need of each case. For the purpose of this research, however, only the cases with 3, 6, and 12 months' postoperative follow-up were taken into account.

The data evaluated corresponded to the primary rhinoplasties of the researcher. The surgical techniques performed on the nasal tip, on the nasal dorsum, and on the nasal base as well as the D1, D2, D3, and D4 records were evaluated. Preoperative and follow-up photos at 12 months (D4) were analyzed objectively, and the measurements were compared with the ideal aesthetic standard of a Caucasian nose. All the photos were taken by the same researcher with the same camera and standardization: model Sony Cyber shot DSCW125 7.2 Megapixels, 6.0 (Sony, Japan) fixed zoom at a distance of $1.5 \mathrm{~m}$ between the camera and the volunteer to provide uniformity for the purpose of scale and measurements. The positions were anteroposterior and direct profile.

Inclusion criteria were primary rhinoplasties, Caucasian noses, and age from 15 to 55 years. Exclusion criteria were previous surgical interventions on the nose and on the face, non-Caucasian noses (Mestizo, Asian, Negroid), and patients who did not return for follow-up.

The quantitative evaluation of the anthropometric measurements of the nose was performed by comparing preoperative and 12-month follow-up (D4) photos. The following measurements were analyzed and compared with the ideal aesthetic standard $^{6,7}$ : front view (anteroposterior; - Fig. 1): (1) intercanthal distance, (2) interalar distance; side view (direct profile; - Fig. 2): (3) nasolabial angle, (4) nasal tip projection (through Goode's method; - Fig. 3), and (5) nasofrontal angle. 
According to the nature of the analyzed data, Student $t$ test and the standard deviation test were applied. The level of significance adopted was $p<0.05$.

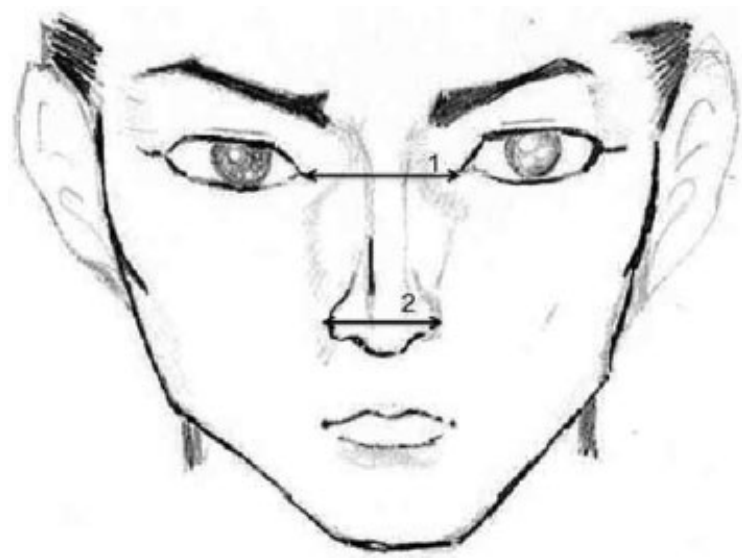

Fig. 1 Front view (anteroposterior). 1, Intercanthal distance; 2, interalar distance.

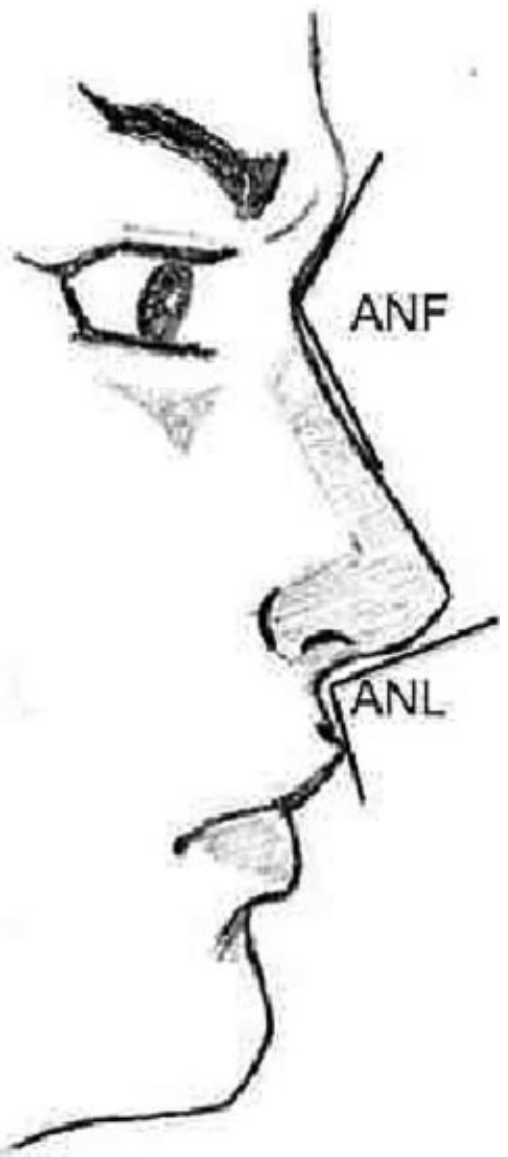

Fig. 2 Side view (direct profile). Abbreviations: ANF, nasofrontal angle; ANL, nasolabial angle.

\section{Results}

Main Techniques Used in Rhinoplasty

Access (Approach)

The predominant access to the nose was endonasal (94.4\%). The closed access without inferior lateral cartilage exposure was predominant in $92.63 \%$ of cases against $7.37 \%$ with delivery.

\section{Surgical Techniques on the Nasal Dorsum}

Removal of osteocartilaginous hump was the most frequent surgical technique performed on the nasal dorsum (33.33\%). Removal of the nasal hump did not happen in all the cases. Release of the superior lateral cartilage was not systematically performed. It was necessary to use grafts in some noses, which featured a change in the type of nose to be operated-in other words, not all the noses needed a reduction operation (-Fig. 4). Of the grafts, $41.94 \%$ of cases had camouflage type added (onlay graft), followed by the spreader graft, which was

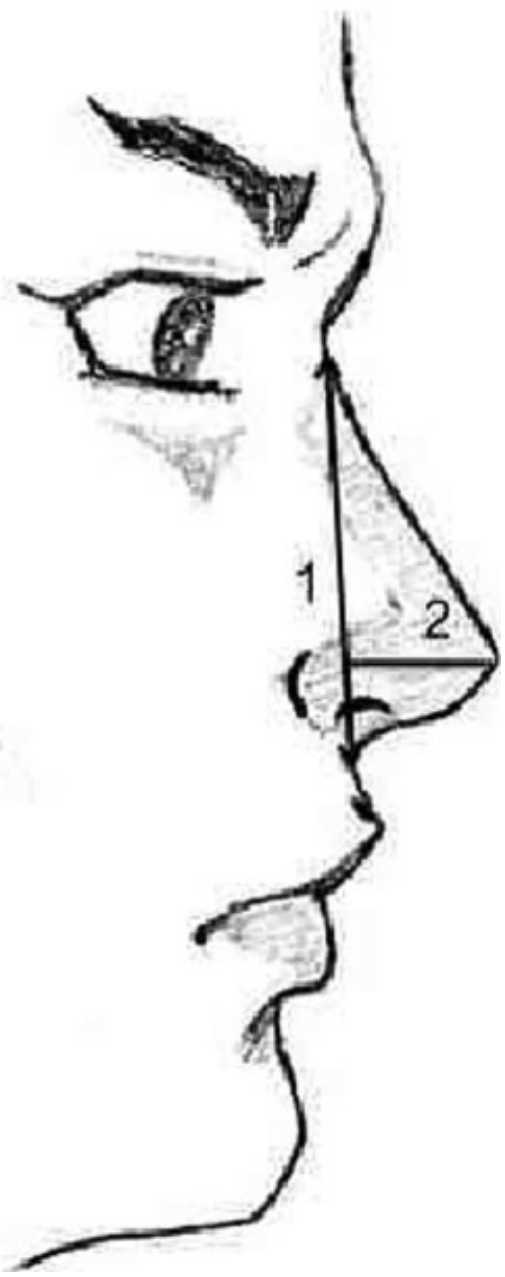

Fig. 3 Side view (direct profile). Note: Measurement of the nasal tip projection through Goode's method, which entails measurement of the perpendicular line from the tip point (1) to the line of the facial plane (2) divided by the measurement of the line from the nasion to the tip point. 
used in $35.48 \%$ of cases (- Fig. 5). In $60.67 \%$ of cases, a lateral osteotomy was performed, followed by paramedian osteotomy in $15.33 \%$ of cases (-Fig. 6). Cartilage was the most frequent graft donor site, with $90.32 \%$ of cases (-Fig. 7); among them, the septal graft showed the highest frequency at 82.14\% (-Fig. 8).

\section{Surgical Techniques on the Nasal Tip}

The use of sutures on the tip (24.76\%) for definition and refinement was the most frequent, followed by La Garde maneuver (19.44\%) for detachment and adjustment of the

\section{Dorsum}

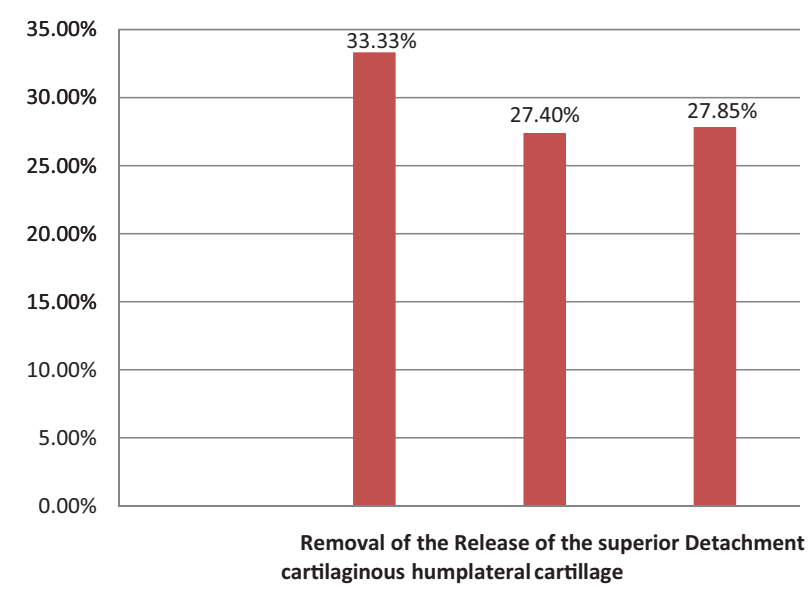

Fig. 4 Surgical maneuvers on the nasal dorsum.

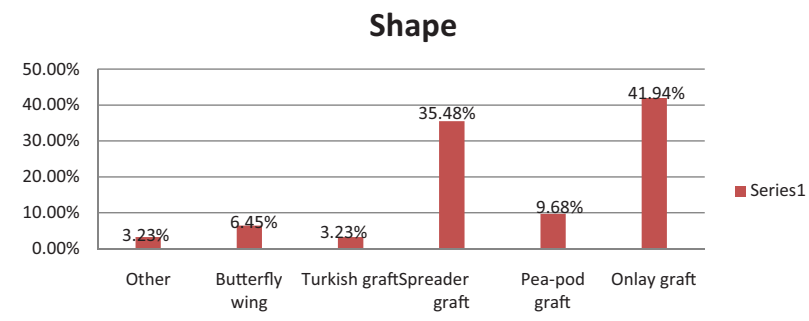

Fig. 5 Surgical maneuvers on the nasal dorsum-grafts.

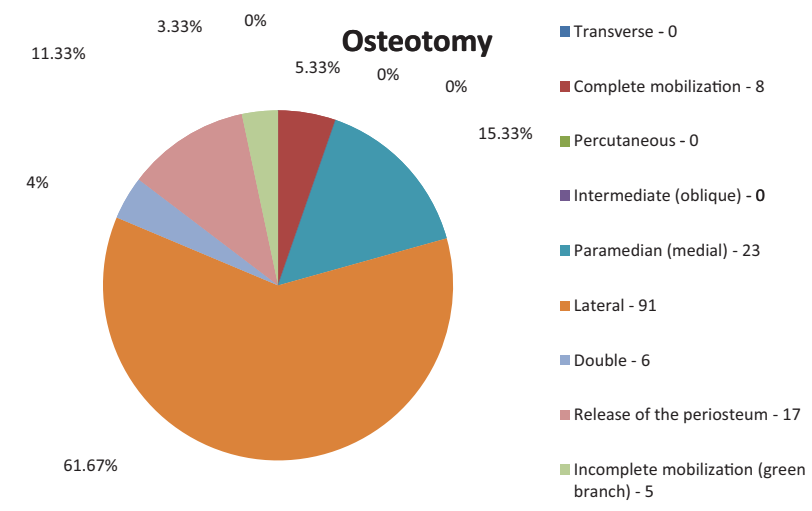

Fig. 6 Surgical maneuvers on the nasal dorsum-osteotomies. skin; then the resection of the membranous septum (15.67\%) for cephalic rotation, the placement of grafts (10.66\%) for structure and refinement, and then the interposing maneuver between the septum and the medial crura (tongue-in-groove; 9.72\%) for support of the tip. Cephalic resection of the inferior lateral cartilage occurred in only $5.02 \%$ of cases.

Resection of the membranous septum (15.67\%) was twice as frequent as caudal septum shortening (6.27\%), which confirmed the lesser need of cartilaginous removal from the tip of the noses (-Fig. 9).

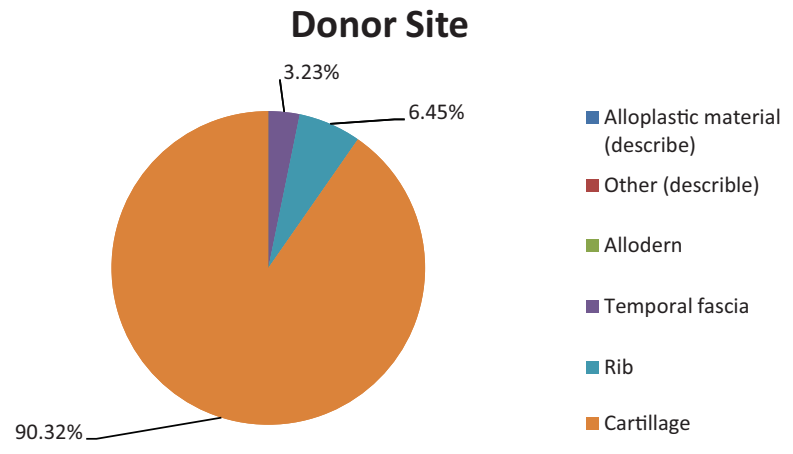

Fig. 7 Surgical maneuvers on the nasal dorsum-graft donor site.

\section{Cartilage}

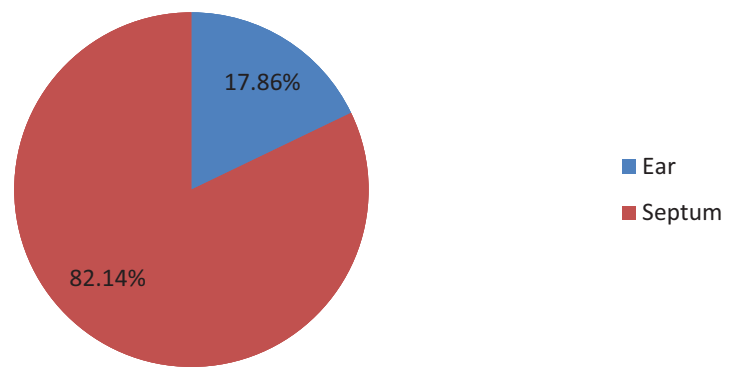

Fig. 8 Surgical maneuvers on the nasal dorsum-graft donor site cartilage.

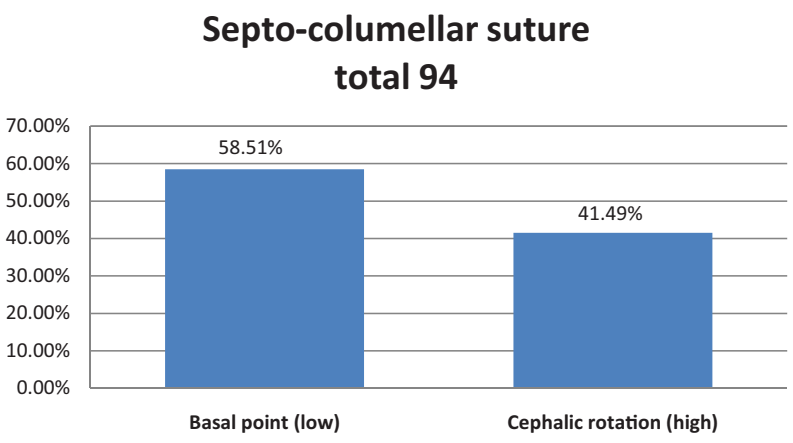

Fig. 9 Surgical maneuvers on the tip of the nose. 


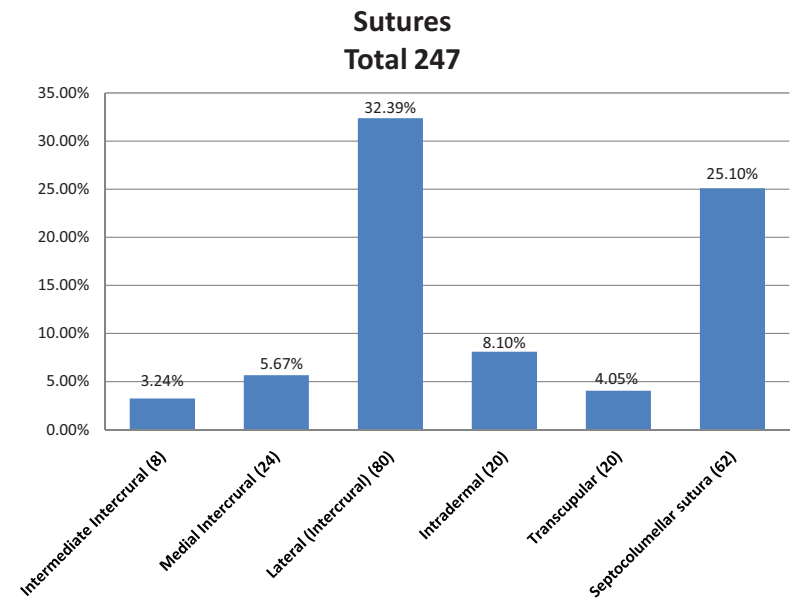

Fig. 10 Surgical maneuvers on the tip of the nose-sutures.

The lateral intercrural was the most frequent suture on the nasal tip, used in $32.39 \%$ of cases. Next was the septocolumellar suture at 25.1\% (-Figs. 10 and 11). The highest frequency of the basal septocolumellar suture (low) was $58.51 \%$, followed by the rotation septocolumellar suture (high), which was observed in $41.49 \%$ of cases.

The type of suture thread used in the surgical technique on the nasal tip is shown in - Fig. 12, with the polydioxanone suture 4-0 being used in the majority of cases (70\%).

The columellar strut was the most frequent graft added to the nasal tip (56.86\%), with the cartilage of the nasal septum the most often used (81.08\%).

Cephalic resection of the inferior lateral cartilage was predominantly performed through McIndoe eversion technique or retrograde $(70 \%)$.

\section{Surgical Techniques on the Nasal Base}

The three areas to be observed in the operation of the nasal base are: (1) the nostrils (nasal vestibule), (2) the distance from the nasal base in relation to the intercanthal distance, and (3) the alar-flare, in other words, the external outline of the lateral wall of the inferior lateral cartilage right above the facial groove.

More than one technique can be performed on the same patient depending on the alterations found. The most common techniques used were the correction of asymmetric

\section{Septocollumelar suture Total 94}

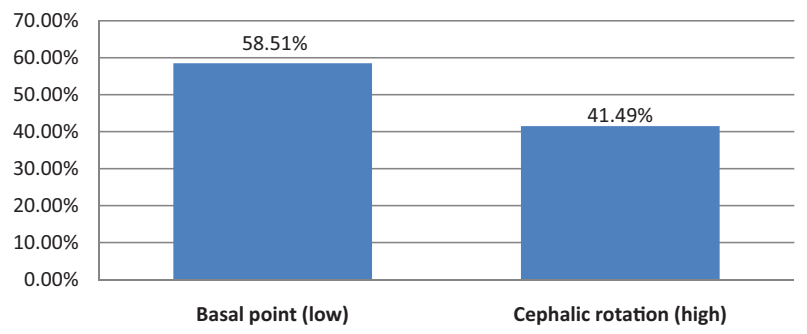

Fig. 11 Surgical maneuvers on the tip of the nose-medial intercrural suture.

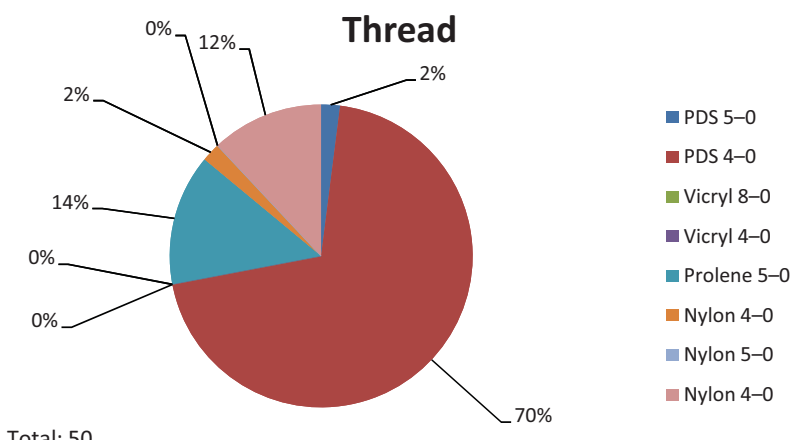

Fig. 12 Surgical maneuvers on the tip of the nose-threads.

nostrils (28.38\%), followed by cerclage (25.68\%) to reduce the alar flare, and resection of the alar base (22.97\%) to reduce the width of the nasal base. For the internal correction of the flaring of the lateral inferior wall, the technique of Cinelli was used in $10.81 \%$ of cases. Release of the periosteum from the premaxilla (1.35\%) and alar wall debulking (1.35\%) were performed in only one patient. It was not necessary to perform larger operations such as the V-Y advancement $(0 \%)$ in any of the patients. There was no incidence of dermabrasion, a procedure to reduce scars (-Fig. 13).

The modified Weir's technique for the resection of the alar base was the most frequent (62.5\%; - Fig. 14).

In the cerclage of the nasal base, the most commonly used thread was Mononylon 4-0 (83.33\%) (Johnson \& Johnson Medical, São Paulo, Brazil).

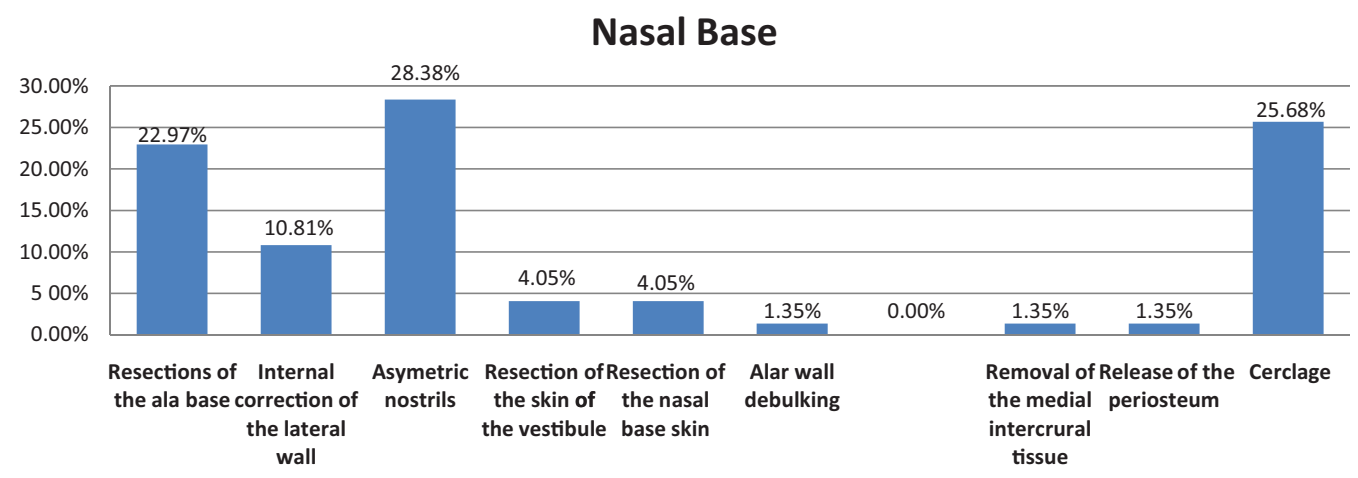

Fig. 13 Surgical maneuvers on the nasal base. 


\section{Resection of the base}

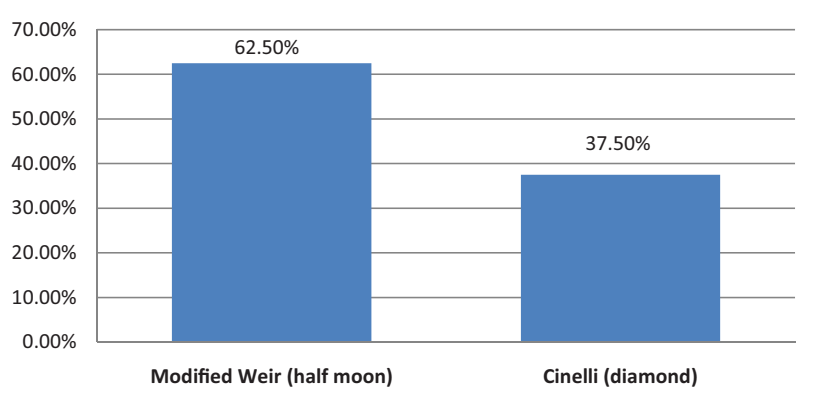

Fig. 14 Surgical maneuvers on the nasal base-resection of the alar base.

One hundred twelve patients returned in 12 months for follow-up and participated in all the phases of the study. Fiftyeight patients did not complete the study.

\section{Comparative Study of the Preoperative and Postoperative Anthropometric Measurements of the Nose at 12-Month Follow-Up}

\section{Nasal Tip Projection}

Goode's method was used through the measurement of the perpendicular line from the tip point to the line of the facial plane divided by the measurement of the line from the nasion to the tip point ( - Fig. 3 ). It is recognized that the value should be from 0.55 to 0.66 . We found an increase in the ratio in both sexes (-Table $\mathbf{1}$ ). There are significant differences in the averages of the projection coefficient between preoperative and postoperative phases in the total group and in both male and female subjects.

\section{Nasolabial Angle}

The nasolabial angle is the angular inclination of the columella at the point where it meets the superior lip (-Fig. 2). A nasolabial angle that varies between 90 and 120 degrees is considered ideal. The average found for the nasolabial angle was higher for both sexes ( $\mathbf{-}$ Table $\mathbf{2}$ ).

There were significant differences in the averages of the nasolabial angle between preoperative and postoperative phases in the total groups and in male and female patients.

\section{Nasofrontal Angle}

The nasofrontal angle is found by tracing a tangent line to the glabella through the nasion, which crosses a line traced tangent to the nasal dorsum (-Fig. 2). The ideal measurement varies between 115 and 130 degrees. We found an increase in the angle in the male sex and a decrease in the angle of the female sex (-Table 3 ). The differences of the nasofrontal angle averages between preoperative and postoperative phases in the total group and in male and female patients were significant.

Table 1 Preoperative and 12-month postoperative projection coefficient

\begin{tabular}{|c|c|c|c|c|c|}
\hline \multirow[t]{2}{*}{ Group/surgical phase } & \multirow[t]{2}{*}{$n$} & \multicolumn{3}{|c|}{ Projection coefficient } & \multirow[t]{2}{*}{$p$} \\
\hline & & Min-max & Average & sd & \\
\hline Total/pre & 112 & $0.60-0.68$ & 0.63 & 0.02 & 0.00001 \\
\hline Total/post & 112 & $0.61-0.68$ & 0.65 & 0.01 & \\
\hline Female/pre & 75 & $0.61-0.68$ & 0.63 & 0.02 & 0.00001 \\
\hline Female/post & 75 & $0.61-0.68$ & 0.65 & 0.02 & \\
\hline Male/pre & 37 & $0.60-0.68$ & 0.63 & 0.02 & 0.000001 \\
\hline Male/post & 37 & $0.62-0.68$ & 0.65 & 0.01 & \\
\hline
\end{tabular}

Abbreviations: min-max, minimum and maximum value; post, postsurgical; pre, presurgical; sd, standard deviation.

Table 2 Preoperative and 12-month postoperative nasolabial angle

\begin{tabular}{|c|c|c|c|c|c|}
\hline \multirow[t]{2}{*}{ Group/surgical phase } & \multirow[t]{2}{*}{$n$} & \multicolumn{3}{|c|}{ Nasolabial angle } & \multirow[t]{2}{*}{$p$} \\
\hline & & Min-max & Average & sd & \\
\hline Total/pre & 112 & $65.3-138.5$ & 97.50 & 12.86 & $<0.0001$ \\
\hline Total/post & 112 & $80.3-120.9$ & 102.77 & 9.41 & \\
\hline Female/pre & 75 & $78.5-126.2$ & 98.73 & 11.38 & $<0.0001$ \\
\hline Female/post & 75 & $80.3-120.9$ & 104.08 & 9.16 & \\
\hline Male/pre & 37 & $65.3-138.5$ & 95.00 & 15.30 & \\
\hline Male/post & 37 & $80.4-120.3$ & 100.10 & 9.48 & \\
\hline
\end{tabular}

Abbreviations: min-max, minimum and maximum values; post, postsurgical; pre, presurgical; sd, standard deviation. 
Table 3 Preoperative and 12-month postoperative nasofrontal angle

\begin{tabular}{|c|c|c|c|c|c|}
\hline \multirow[t]{2}{*}{ Group/surgical phase } & \multirow[b]{2}{*}{$n$} & \multicolumn{2}{|c|}{ Nasofrontal angle } & \multirow[b]{2}{*}{ sd } & \multirow[t]{2}{*}{$p$} \\
\hline & & Min-max & Average & & \\
\hline Total/pre & 112 & $105.4-159.4$ & 134.29 & 8.77 & 0.008 \\
\hline Total/post & 112 & $120.3-142.2$ & 132.24 & 3.61 & \\
\hline Female/pre & 75 & $111.9-159.4$ & 135.71 & 7.28 & 0.00002 \\
\hline Female/post & 75 & $125.0-142.2$ & 132.31 & 3.38 & \\
\hline Male/pre & 37 & $105.4-149.1$ & 131.41 & 10.75 & 0.67 \\
\hline Male/post & 37 & $120.3-139.2$ & 132.11 & 4.09 & \\
\hline
\end{tabular}

Abbreviations: min-max, minimum and maximum value; post, postsurgical; pre, presurgical; sd, standard deviation.

\section{Discussion}

In a Brazilian journal, Patrocínio et al showed the importance of the surgical record and the knowledge of the surgical techniques performed in rhinoplasty. 8 In our study, we recorded all the surgical techniques performed and we evaluated the most frequently used ones on the nasal dorsum, on the nasal tip, and on the nasal base. Because noses in our region are mostly Caucasian, which were the aim of our study, our results are different regarding the accesses, the types of grafts on the nasal tip, and the frequency of surgery on the nasal base compared with the findings by Patrocínio et al. ${ }^{8}$

The findings regarding the evolution of patients made it possible for us to identify the best techniques to achieve excellence in our results. The postoperative follow-up of the patient is essential for the analysis of the results; the doctorpatient relationship is important even in the presence of unsatisfactory results.

Standardized photographic documentation is essential; preoperative and postoperative photos serve to document the results.

The scarring process of the nose is directly related to the surgical trauma, to the type of skin, the type of cartilage, and postoperative care.

International magazines on facial plastic surgery advise the need to restrict qualitative evaluations and to increase quantitative evaluations more objectively with the use of tools such as the Rhinoplasty Outcomes Evaluation. ${ }^{9}$

Nasal measurements, despite providing objective data of the evaluation, are not more important than the patient's satisfaction. Both pieces of information are important for the patient's postoperative follow-up.

According to a study performed by McKiernan et al in $2001,{ }^{10}$ the impact of rhinoplasty in the quality of life can be evaluated through tools such as the Glasgow Inventory. In the last decade, the quality of life has been a common matter of discussion, and rhinoplasty has a highly positive impact on the functional purpose as well as on the aesthetic one.

Leong and White stated that the interalar and intercanthal ratio is calculated through the division of the interalar distance by the intercanthal distance ( $\mathbf{- F i g . ~ 1 ) . 6}$ The ideal interalar-to-intercanthal ratio of the Caucasian nose is considered as 1 , that is $100 \%$ equivalent ( - Table 4 ). In our study, the average postoperative proportion in the male group, the female group, and total group was lower when compared with the same group preoperatively $(p>0.05)$, approaching the ideal values.

According to Goode's method, the ideal nasal projection is a 0.67 ratio. In our study, we observed increased nasal projection at 12 months postoperatively $(p<0.05)$, being closer to the aesthetic ideal of the nose.

Several authors stated that the ideal nasolabial angle should be found between 90 and 120 degrees, but some authors suggest that it should be kept between 90 and 105 degrees. It is a consensus, however, that the measurement of the male nose should have a more angle and the female nose should have a more obtuse angle, promoting higher rotation on the nasal tip in women. ${ }^{11-14}$

In a population study in the city of Dundee, Scotland, Leong and White found a value between 67 and 116 degrees. ${ }^{6}$ In our

Table 4 Preoperative and 12-month postoperative interalar and intercanthal distances

\begin{tabular}{|c|c|c|c|c|c|}
\hline \multirow[b]{2}{*}{ Group/surgical phase } & \multirow[b]{2}{*}{$n$} & \multicolumn{3}{|c|}{ Proportion of interalar and intercanthal distances } & \multirow[b]{2}{*}{$p$} \\
\hline & & Min-max & Average & sd & \\
\hline Total/pre & 112 & $0.75-1.58$ & 1.12 & 0.15 & 0.00004 \\
\hline Total/post & 112 & $0.75-1.42$ & 1.08 & 0.13 & \\
\hline Female/pre & 75 & $0.81-1.55$ & 1.10 & 0.15 & 0.002 \\
\hline Female/post & 75 & $0.81-1.41$ & 1.07 & 0.12 & \\
\hline Male/pre & 37 & $0.75-1.58$ & 1.15 & 0.16 & 0.009 \\
\hline Male/post & 37 & $0.75-1.42$ & 1.09 & 0.14 & \\
\hline
\end{tabular}

Abbreviations: min-max, minimum and maximum value; post, postsurgical; pre, presurgical; sd, standard deviation. 
study, the average of value was $97.50 \pm 12.86$ preoperatively and $102.77 \pm 9.41$ postoperatively $(p<0.05)$, showing an increase in the nasolabial angle and in conformity with the ideal aesthetic values for a Caucasian nose.

In our study, the average nasofrontal angle postoperatively in the female group was lower than the average preoperative value ( $p>0.05$ ), which probably occurred due to the reduction of the nasal dorsum through surgical maneuvers such as the removal of the osteocartilaginous hump, approaching the ideal values for the Caucasian nose mentioned by Leong and White. 6

It is important to remember that the facial static morphology, despite being the dominant factor in the aesthetic criteria, is not the only factor to be considered. Therefore, dynamic proportions, skin texture, and color as well as the appearance of teeth are also important in facial aesthetic. ${ }^{7}$ For that reason, a multidisciplinary team becomes necessary with the aid of dermatologists, orthodontists, and bucomaxillofacial surgeons, among others. In addition, in the final evaluation, more than the standard aesthetic measurements, the most important thing is that the patient's desire in sync with the surgeon's aesthetic sense.

The learning curve is based on the development of tactile skills and on the surgeon's judgment. Surgical success is based on the systematization of surgical steps, the level of search for excellence of new technical details, and surgical maneuvers that are being improved, through sutures and grafts, always striving for the best refinement for the Caucasian nose.

This study begins the research of the records of surgical maneuvers in rhinoplasty and of the standardized objective evaluations of the surgical results at the hospital.

\section{Conclusion}

The main surgical techniques in rhinoplasty on Caucasian noses were studied and are currently vast. The anthropometric evaluation at 12-month follow-up of the nose showed quantitatively the efficacy of the procedures performed when compared with the ideal aesthetic measurements for a Caucasian nose.

\section{References}

1 Gunter J. Dallas Rhinoplasty: Nasal Surgery by the Masters. St. Louis: Quality Medical Publishing; 2006:2279

2 Pinto JSP. Interface de visibilização de informações para o sistema integrado de protocolos eletrônicos [Tese]. Paraná, Brazil: Universidade Federal do Paraná, Setor de Ciências da Saúde; 2005:111

3 Maniglia AJ, Maniglia JJ, Maniglia JV. Rinoplastia Estética Funcional e Reconstrutora. São Paulo, Brazil: Revinter; 2002:129-50

4 Tardy M, Brown RJ. Surgical Anatomy of the Nose. New York, NY: Raven Press; 1990:12-66

5 Berger C. Electronic data collection for the analysis of surgical maneuvers on patients submitted to rhinoplasty. Int Arch Otorhinolaryngol 2012;16(4):497-501

6 Leong SCL, White PS. A comparison of aesthetic proportions between the healthy Caucasian nose and the aesthetic ideal. J Plast Reconstr Aesthet Surg 2006;59(3):248-252

7 Farkas LG, Katic MJ, Forrest CR, et al. International anthropometric study of facial morphology in various ethnic groups/races. J Craniofac Surg 2005;16(4):615-646

8 Patrocínio LG, Carvalho PM, de Souza HM, Couto HG, Patrocínio JA. Surgical maneuvers performed on rhinoplasty procedures carried out at an otorhinolaryngology residency program. Braz J Otorhinolaryngol 2006;72(4):439-442

9 Luce EA. Outcome studies and practice guidelines in plastic surgery [editorial]. Plast Reconstr Surg 1999;104(4):1187-1190

10 McKiernan DC, Banfield G, Kumar R, Hinton AE. Patient benefit from functional and cosmetic rhinoplasty. Clin Otolaryngol Allied Sci 2001;26(1):50-52

11 Doddi NM, Eccles R. The role of anthropometric measurements in nasal surgery and research: a systematic review. Clin Otolaryngol 2010;35(4):277-283

12 Piccardi GH. Assessing facial beauty through proportion analysis by image processing and supervised learning. Int J Hum Comput Stud 2006;64(12):1184-1199

13 Gruber RP, Weintraub J, Pomerantz J. Suture techniques for the nasal tip. Aesthet Surg J 2008;28(1):92-100

14 Guyuron B, Behmand RA. Nasal tip sutures part II: the interplays. Plast Reconstr Surg 2003;112(4):1130-1145, discussion 11461149 